BMJ Open

Diabetes

Research

\& Care

\section{Association of G6PD variants with hemoglobin A1c and impact on diabetes diagnosis in East Asian individuals}

To cite: Leong A, Lim VJY, Wang $C$, et al. Association of $G 6 P D$ variants with hemoglobin A1c and impact on diabetes diagnosis in East Asian individuals. BMJ Open Diab Res Care 2020;8:e001091. doi:10.1136/ bmjdrc-2019-001091

- Additional material is published online only. To view please visit the journal online (http://dx.doi.org/10.1136/ bmjdrc-2019-001091).

AL and VJYL contributed equally.

Received 2 December 2019 Revised 20 January 2020 Accepted 14 February 2020

Check for updates

C A Author(s) (or their employer(s)) 2020. Re-use permitted under CC BY-NC. No commercial re-use. See rights and permissions. Published by BMJ.

For numbered affiliations see end of article.

Correspondence to

Dr Xueling Sim;

ephsx@nus.edu.sg and Dr

Aaron Leong:

asleong@partners.org

\section{ABSTRACT}

Objective Hemoglobin A1c (HbA1c) accuracy is important for diabetes diagnosis and estimation of overall glycemia. The G6PD-Asahi variant which causes glucose-6phosphate dehydrogenase (G6PD) deficiency has been shown to lower $\mathrm{HbA1c}$ independently of glycemia in African ancestry populations. As different G6PD variants occur in Asian ancestry, we sought to identify Asianspecific G6PD variants associated with $\mathrm{HbA1c}$.

Research design and methods In eight Asian population-based cohorts, we performed imputation on the $X$ chromosome using the 1000 Genomes reference panel and tested for association with $\mathrm{HbA1c}(10005$ East Asians and 2051 South Asians). Results were meta-analyzed across studies. We compared the proportion of individuals classified as having diabetes/pre-diabetes by fasting glucose $\geq 100 \mathrm{mg} / \mathrm{dL}$ or $\mathrm{HbA} 1 \mathrm{c} \geq 5.7 \%$ units among carriers and non-carriers of $\mathrm{HbA} 1 \mathrm{c}$-associated variants.

Results The strongest association was a missense variant (G6PD-Canton, rs72554665, minor allele frequency $=2.2 \%$, effect in men $=-0.76 \%$ unit, $95 \% \mathrm{Cl}-0.88$ to -0.64 , $\mathrm{p}=1.25 \times 10^{-27}, \mathrm{n}=2844$ ). Conditional analyses identified a secondary distinct signal, missense variant (G6PDKaiping, rs72554664, minor allele frequency $=1.6 \%$, effect in men $=-1.12 \%$ unit, $95 \% \mathrm{Cl}-1.32$ to -0.92 , $\left.\mathrm{p}=3.12 \times 10^{-15}, \mathrm{p}_{\text {conditional Canton }}=7.57 \times 10^{-11}\right)$. Adjusting for glucose did not attenuate their effects. The proportion of individuals with fasting glucose $\geq 100 \mathrm{mg} / \mathrm{dL}$ did not differ by carrier status of G6PD-Canton ( $p=0.21$ ). Whereas the proportion of individuals with $\mathrm{HbA} 1 \mathrm{C} \geq 5.7 \%$ units was lower in carriers (5\%) compared with non-carriers of G6PD-Canton $(30 \%, p=0.03)$.

Conclusions We identified two G6PD variants in East Asian men associated with non-glycemic lowering of $\mathrm{HbA1c}$. Carriers of these variants are more likely to be underdiagnosed for diabetes or pre-diabetes than noncarriers if screened by HbA1c without confirmation by direct glucose measurements.

\section{INTRODUCTION}

Hemoglobin A1c (HbA1c), a biomarker that reflects average glycemia over the previous 3 months, ${ }^{1}$ is routinely used to estimate glycemic control in patients with diabetes, and more

\section{Significance of this study}

What is already known about this subject?

- Hemoglobin A1c ( $\mathrm{HbA1c}$ ) accuracy is important for diabetes classification and estimation of overall glycemia.

- A recent genome-wide association study metaanalysis of $\mathrm{HbA1C}$ identified an African-specific missense variant in G6PD (Asahi rs1050828) that lowered $\mathrm{HbA1c}$ in African-Americans independently of glycemia. Carriers of this variant were likely to be underdiagnosed for diabetes if $\mathrm{HbA1c}$ were used as the sole diagnostic criterion.

- G6PD variants are diverse globally and occur quite commonly in Asia; yet, the effects of Asian-specific $G 6 P D$ variants on $\mathrm{HbA} 1 \mathrm{c}$ remain unclear.

What are the new findings?

- Through association analysis of the $X$ chromosome with $\mathrm{HbA} 1 \mathrm{C}$ in 12056 Asians, we identified associations of two distinct low-frequency variants that lowered $\mathrm{HbA} 1 \mathrm{C}$ independently of glycemia, G6PD-Canton (rs72554665, effect in men $=-0.76 \%$ unit, $95 \% \mathrm{Cl}-0.88$ to -0.64 ) and G6PD-Kaiping (rs72554664, effect in men $=-1.12 \%$ unit, $95 \% \mathrm{Cl}$ -1.32 to -0.92 ).

- Carriers of these Asian-specific G6PD variants are more likely to be underdiagnosed for diabetes or pre-diabetes than non-carriers if they are screened by $\mathrm{HbA1C}$ without confirmation by direct glucose measurements.

How might these results change the focus of research or clinical practice?

- The use of both fasting glucose and $\mathrm{HbA1c}$ in combination for diabetes diagnosis is paramount when screening ethnically diverse populations.

recently, diagnose diabetes. ${ }^{2}$ However, HbA1c can also be influenced by non-glycemic factors, including genetic variants that affect erythrocyte turnover. ${ }^{34}$ A number of genomewide association study (GWAS) meta-analyses 
have identified African-specific variants in G6PD that are associated with HbAlc in African-American and Hispanic individuals in the USA. ${ }^{356}$ One of these is a missense variant (G6PD-Asahi, rs1050828, minor allele frequency $(\mathrm{MAF})=11 \%$ in African-Americans) that lowers HbAlc by $0.81 \%$ ( $95 \%$ CI -0.96 to $-0.66,-8.9 \mathrm{mmol} / \mathrm{mol}, 95 \%$ CI -10.6 to $\left.-7.2, p=8.23 \times 10^{-135}\right),{ }^{3}$ and causes glucose6-phosphate dehydrogenase (G6PD) deficiency, an X linked disease characterized by hemolysis in response to infections, and certain foods or drugs. ${ }^{7}$

G6PD deficiency is widespread across malaria-endemic countries with a global prevalence of $4.9 \% .^{8}$ While subSaharan African and the Arabian Peninsula have higher prevalence estimates of the disease (up to 32.5\%) compared with Central and Southeast Asia (less than $20 \%$ ), the majority of G6PD-deficient individuals reside in Asia. ${ }^{9}$ Furthermore, the genetic diversity of $G 6 P D$ variants varies globally, exhibiting high heterogeneity in Asia-Pacific regions. ${ }^{10}$ While several G6PD variants have been reported to occur in Asia, their effects on HbAlc and implications on the clinical utility of $\mathrm{HbAlc}$ remain unclear. Notably, many Asian countries have adopted the standard threshold of $\mathrm{HbAlc} \geq 6.5 \%(48 \mathrm{mmol} / \mathrm{mol})$ to diagnose diabetes. ${ }^{211}$

We hypothesized that G6PD variants occurring in Asian ancestry individuals affect the diagnostic accuracy of HbA1c. To our knowledge, previous GWAS to identify HbA1c-associated genetic variants included relatively smaller samples of Asian ancestry, and excluded analysis of the $\mathrm{X}$ chromosome due to its non-diploid nature and lack of analytical methods, limiting the discovery of Asianspecific genetic variants. Here, to discover Asian-specific G6PD variants, we performed imputation on the chromosome $\mathrm{X}(\mathrm{ChrX})$ with the 1000 Genomes reference panel and tested the association of variants with $\mathrm{HbAlc}$ in eight Asian studies.

\section{RESEARCH DESIGN AND METHODS}

\section{Cohort studies included in meta-analysis}

We included genome-wide array data from the following eight studies: Multi-Ethnic Cohort (MEC), Singapore Prospective Study Program (SP2), Living Biobank, Singapore Malay Eye Study (SiMES), the Singapore Chinese Eye Study (SCES), Singapore Indian Eye Study (SINDI), Singapore Chinese Health Study (SCHS-MI), and Beijing Eye Study (BES, online supplementary table 1 and online supplementary appendix).

\section{Glycemic trait measurements and exclusion criteria}

We excluded individuals with self-reported physiciandiagnosed diabetes, use of diabetes medication, or undiagnosed diabetes (fasting glucose (FG) $\geq 126 \mathrm{mg} / \mathrm{dL}$ ( 7 $\mathrm{mmol} / \mathrm{L})$ or random glucose $(\mathrm{RG}) \geq 200 \mathrm{mg} / \mathrm{dL}(11.1$ $\mathrm{mmol} / \mathrm{L})$ or $\mathrm{HbAlc} \geq 6.5 \%(48 \mathrm{mmol} / \mathrm{mol})$ where available). FG was measured after an overnight 8-12 hours' fast using enzymatic methods (ADVIA 2400; Bayer Diagnostics and Siemens Healthcare Diagnostics). HbA1c was reported in National Glycohemoglobin Standardization Program (NGSP) percent.

\section{ChrX array genotype calling, quality control and imputation}

We performed quality control (QC) on ChrX genotypes within each array and only considered the samples that passed autosomal QC. We performed reclustering and genotype calling on the intensity files using GenomeStudio V.2.0. Variants on pseudoautosomal region (PAR) were called from clusters generated by females and males. Those on non-PAR were called from female-only generated clusters. We mapped variants to the hg19 reference genome, forward stand, and excluded those that were unmapped, had a call rate $<0.95$, or a Hardy-Weinberg equilibrium $\mathrm{p}$ value $<10^{-6}$. We further excluded samples with $>5 \%$ missingness on $\mathrm{ChrX}$ or chromosome $\mathrm{Y}$ or if the reported sex did not match the genetic sex.

A second round of variant $Q C$ was performed to ensure that physical positions were matched to the 1000 Genomes Phase 3 (1000G) reference panel, ${ }^{12}$ alleles were on the forward strand, palindromic variants with MAF $>0.4$ were excluded, and allele frequencies of Chinese and Indians were within 0.2 from 1000G East Asians (EAS) and South Asians (SAS), respectively, and Malays were within 0.3 from 1000G EAS (online supplementary table 2). The final set of ChrX variants was prephased using SHAPEITv2. ${ }^{13}$ Heterozygous haploid variants in males were set as missing and reimputed. The phased genotypes were then imputed using Michigan Imputation Server ${ }^{14}$ with the full $1000 \mathrm{G}$ Phase $3 v 5$ as the reference panel.

\section{Accuracy of ChrX data imputation}

To maximize sample set by study, we prioritized samples genotyped on the Illumina arrays. We evaluated the accuracy of imputation by checking the concordance in a subset of samples with both imputed genotypes and exome sequencing: SINDI (599 men and 518 women), SP2 (841 men and 1055 women) within the Type 2 Diabetes Genetic Exploration by Next-generation sequencing in multi-Ethnic Samples (T2D-GENES) consortium, ${ }^{15} 16$ and the Living Biobank (1228 men and 1220 women). ${ }^{17}$ The remaining exomes not previously genotyped in T2D-GENES (Chinese: 156 men and 76 women; Indians: 229 men and 336 women) were used for association with HbAlc. Generally, men had higher concordance rate $(>99 \%)$ than women $(>97 \%)$ for all overlapping variants (online supplementary table 3 ). The imputed calls for the two most common G6PD mutations in Singapore, ${ }^{18}$ G6PD-Canton and G6PD-Kaiping, were highly concordant with the exome calls, with $>98.6 \%$ and $100 \%$ concordance for Canton and Kaiping, respectively, in Living Biobank. In SP2, concordance with G6PDCanton was $>98.1 \%$ across the Illumina $1 \mathrm{M}$ and 610 arrays.

\section{Association tests and meta-analysis}

We tested imputed ChrX variants for association with HbAlc by study, in men and women separately. We regressed trait values with age, age $^{2}$ and the first three principal components to obtain residuals. We then 
regressed inverse-normalized residuals against the variants and incorporated empirical kinship matrices using RVTESTS. ${ }^{19}$ We repeated all regression analyses with the untransformed trait values to obtain effect size change.

To combine association results from sex-stratified analyses, we first excluded variants that were poorly imputed (imputation Rsq $<0.3$ ), had a minor allele count (AC) $<5$ or association test $\mathrm{SE}>10$ within sex-stratified analyses. We assumed that non-PAR variants in men were homozygous diploid when combining with women in an additive model. Using METAL, we combined p values from inversenormalized trait values and weighted them by sample size. ${ }^{20}$ We combined effect sizes from untransformed traits using inverse-variance weights. Meta-analyses were performed within each ancestry (EAS: BES, Living Biobank, SCES, SCHS-MI, SiMES, SP2, MEC; SAS: SINDI) in variants that were present in two or more studies. To control for residual population structure, genomic control (GC) was applied at the study level and meta-analysis.

We used $p$ value $<5 \times 10^{-8}$ in the sex-stratified or sexcombined meta-analyses to declare genome-wide significance. Association results were visualized using regional LocusZoom plots. ${ }^{21} \mathrm{QC}$ and analysis workflows of the association and meta-analysis can be found in online supplementary figure 1 . We used $\mathrm{dbNSFP}^{22}$ and the Variant Effect Predictor ${ }^{23}$ to compile function predictions, gene annotations (eg, coding, regulatory, upstream) and consequences (eg, non-sense, missense, intronic) to annotate the variants.

To determine whether associations were independent of glycemia, we performed association testing with (1) FG/RG and (2) HbAlc adjusted for FG/RG where available, using both inverse-normalized traits and raw traits.

\section{Implications of HbA1c-associated variants on undiagnosed diabetes and pre-diabetes classification}

To investigate the impact of $\mathrm{HbAlc}$-associated variants on undiagnosed diabetes and pre-diabetes classification, we first excluded individuals with a prior diagnosis of diabetes or medication use, and then compared the proportion of individuals classified as having pre-diabetes or undiagnosed diabetes by FG across the genotype categories at HbAlc-associated variants. Of the eight studies, only three (SP2, MEC, Living Biobank) measured both HbA1c and FG $(n=2566)$. To classify impaired FG or undiagnosed diabetes, we used the American Diabetes Association (ADA) biochemical criteria of HbAlc $\geq 5.7 \%$ (39 mmol/ $\mathrm{mol})$ or $\mathrm{FG} \geq 100 \mathrm{mg} / \mathrm{dL}(5.6 \mathrm{mmol} / \mathrm{L}),{ }^{2}$ respectively. In a sensitivity analysis, we used the WHO criteria of HbAlc $\geq 6.0 \%$ (42 mmol $/ \mathrm{mol})$ or FG $\geq 110 \mathrm{mg} / \mathrm{dL}(6.1 \mathrm{mmol} / \mathrm{L}) .{ }^{11}$

\section{RESULTS}

Summary of association results on ChrX

We performed a meta-analysis of ChrX association with HbA1c on 10005 EAS and 2051 SAS from eight studies. The GC inflation factors ranged from 0.965 to 1.069 in men and from 0.894 to 1.327 in women (likely due to low sample size in one of the studies). After applying studylevel GC correction, GC inflation factors were less than 1.0 in sex-stratified and sex-combined meta-analyses. Thirty-six variants, all located within $2.2 \mathrm{Mb}$ around $G 6 P D$, reached genome-wide significance in EAS men. None reached genome-wide significance in EAS women (online supplementary table 4 and online supplementary figure 2). In EAS sex-combined meta-analysis, 35 of these 36 variants remained significant. In SAS, none reached genome-wide significance.

\section{Strongest association with $\mathrm{HbA1c}$ at G6PD-Canton in men}

The lead variant in men, rs72554665, was a lowfrequency missense variant, G6PD-Canton $(\mathrm{MAF}=2.2 \%$, $\left.\mathrm{p}=1.25 \times 10^{-27}\right) \quad($ table 1$)$. This variant lowered HbAlc by $-0.76 \%$ (95\% CI -0.88 to -0.64$), 8.3 \mathrm{mmol} / \mathrm{mol}(95 \%$ CI -9.6 to -7.0 ), and explained $6.9 \%$ of the variance in men. In women, G6PD-Canton showed suggestive association $\left(\mathrm{MAF}=1.8 \%, \mathrm{p}=1.40 \times 10^{-7}\right)$; each copy of the minor allele lowered HbA1c by $-0.39 \%$ ( $-4.3 \mathrm{mmol} /$ mol) and accounted for $1.0 \%$ of the variance. The lead variant in women was an intergenic variant, rs148112010 $\left(\mathrm{MAF}=1.6 \%, \mathrm{p}=5.36 \times 10^{-8}\right.$, table 1$)$ which was in moderate linkage disequilibrium (LD) with G6PD-Canton $\left(\mathrm{r}^{2}=0.52\right.$ in 1000G EAS).

In the sex-combined meta-analysis, the lead variant was at ChrX:153734352 $\left(\mathrm{MAF}=2.0 \%, \mathrm{p}=1.71 \times 10^{-30}\right)$, with evidence of heterogeneity between men and women $\left(\mathrm{p}_{\text {het }}=6.7 \times 10^{-4}\right) \quad($ table 1$)$. This variant was in complete LD $\left(r^{2}=1.00\right)$ with G6PD-Canton. Conditioning on G6PDCanton abolished its association ( $p_{\text {conditional_Canton }}=0.86$, table 1). Direction of effect and strength of association of G6PD-Canton were consistent across studies in both men and women (online supplementary figure 3 and online supplementary table 5).

\section{Conditional analysis on G6PD-Canton identified a second distinct signal: G6PD-Kaiping}

To identify distinct signals at G6PD, we conditioned on the strongest signal-G6PD-Canton. At a Bonferroni corrected threshold of $1.40 \times 10^{-5}$ computed from 3576 variants in the $2.5 \mathrm{Mb}$ region centered on G6PD-Canton, 11 variants remained significant in men (online supplementary table 6) and 10 in the sex-combined analyses. In women, none was significant on conditioning on G6PD-Canton.

The lead secondary signal in men and the sex-combined analysis was rs190054725, an intronic variant on $M P P 1$ and located $279 \mathrm{~kb}$ from G6PD-Canton $\left(\mathrm{r}^{2}=0.0001\right.$, $\mathrm{MAF}_{\text {men }}=0.9 \%$, effect conditional_Canton $=-1.33 \%, 95 \%$ CI -1.57 to $-1.09,-14.5 \mathrm{mmol} / \mathrm{mol}, 95 \%$ CI -17.2 to -11.9 , $\mathrm{p}=7.29 \times 10^{-13}, \mathrm{p}_{\text {conditional_Canton }}=1.07 \times 10^{-14}$, online supplementary figure 4). Joint conditional analyses on G6PD-Canton and rs 190054725 abolished all signals $\left(\mathrm{p}_{\text {conditional }}>1.40 \times 10^{-5}\right)$. Of the remaining 10 variants that remained significant in the conditional analysis, one was a missense $G 6 P D$ variant, rs72554664, G6PD-Kaiping $\left(\mathrm{MAF}_{\text {men }}=1.6 \%\right.$, effect ${ }_{\text {conditional }}$ Canton $=-1.12 \%, 95 \%$ CI -1.35 to $-0.88,-12.2 \mathrm{mmol} / \mathrm{mol}, 95 \%$ 


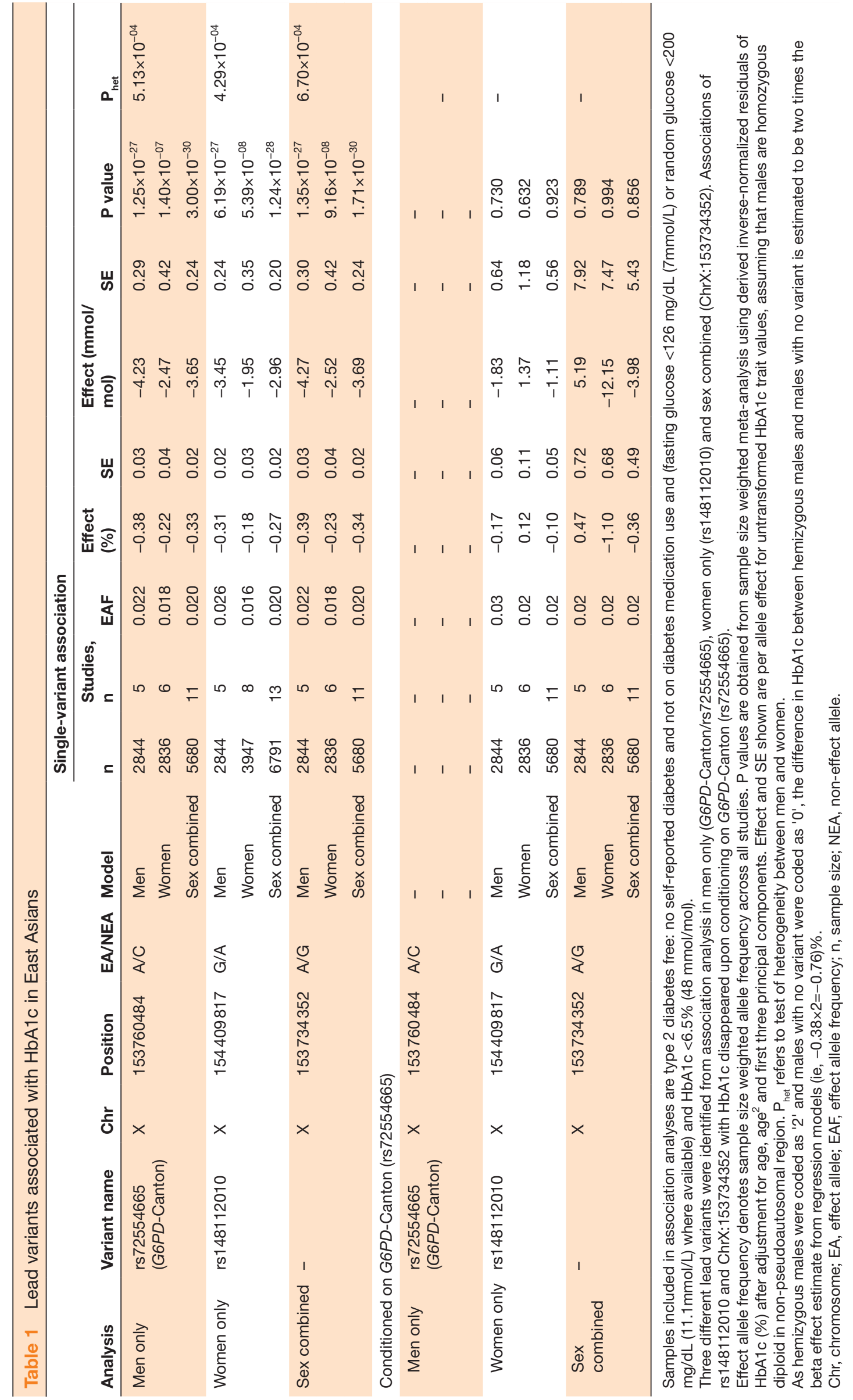

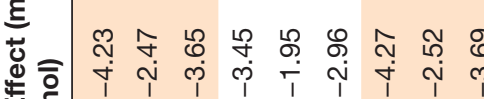

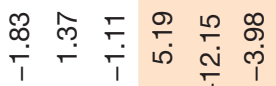
8 당ำ

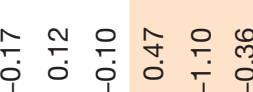

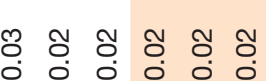

س

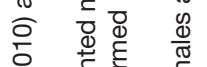

பัల

U

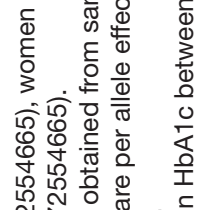

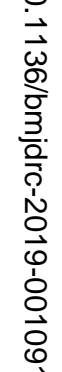

○ 
A

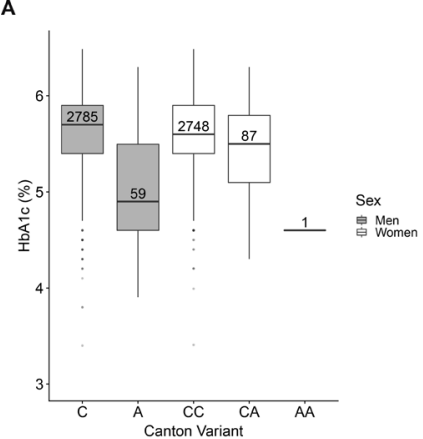

c

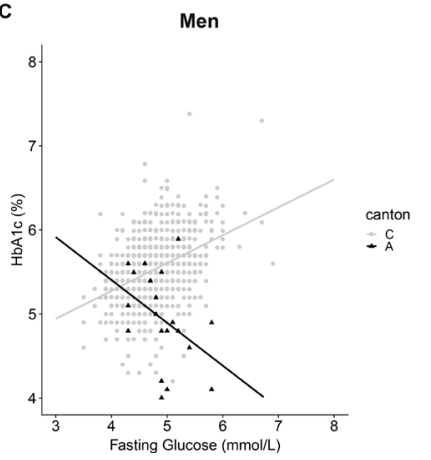

B

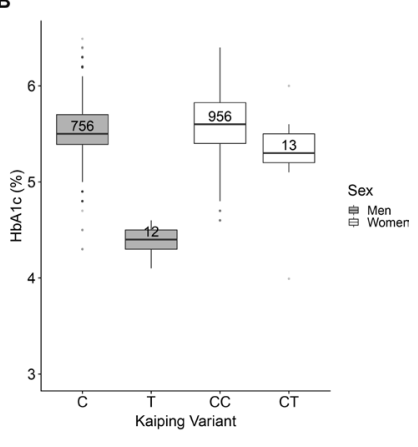

。

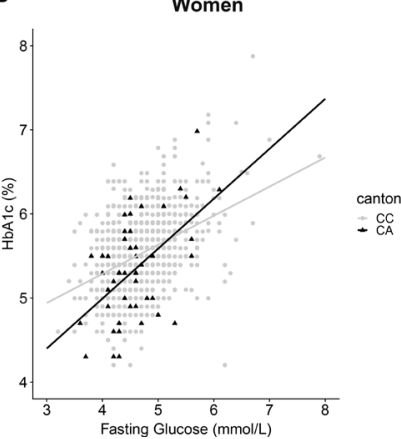

Figure 1 Distribution of $\mathrm{HbA} 1 \mathrm{c}$ (\%) by sex and G6PD variant carrier status, and fasting glucose $(\mathrm{mmol} / \mathrm{L})$. (A) Boxplot of HbA1c by sex and G6PD-Canton (rs72554665). (B) Boxplot of $\mathrm{HbA} 1 \mathrm{c}$ by sex and G6PD-Kaiping (rs72554664). (C) Scatterplot of HbA1c and fasting glucose among men by G6PD-Canton carrier status. (D) Scatterplot of $\mathrm{HbA} 1 \mathrm{c}$ and fasting glucose among women by G6PDCanton carrier status. $\mathrm{HbA} 1 \mathrm{c} 3 \%=9 \mathrm{mmol} / \mathrm{mol} ; 4 \%=20$ $\mathrm{mmol} / \mathrm{mol} ; 5 \%=31 \mathrm{mmol} / \mathrm{mol} ; 6 \%=42 \mathrm{mmol} / \mathrm{mol} ; 7 \%=53$ $\mathrm{mmol} / \mathrm{mol} ; 8 \%=64 \mathrm{mmol} / \mathrm{mol}$. HbA1c, hemoglobin A1c.

CI -14.8 to $-9.6, \mathrm{p}=3.12 \times 10^{-15}, \mathrm{p}_{\text {conditional_Canton }}=7.57 \times 10^{-11}$, online supplementary figure 5 ), which was in moderate LD with rs190054725 $\left(\mathrm{r}^{2}=0.62\right)$. Neither association at the two missense variants attenuated on reciprocal conditioning $\left(r^{2}=0, D^{\prime}=1\right.$ in $1000 \mathrm{G}$ EAS $)$. When conditioned on both G6PD-Kaiping and G6PD-Canton, none of the variants remained significant $(p>0.1$; online supplementary table 7).

\section{Other G6PD variant associations with $\mathrm{HbA1c}$}

We extracted the association statistics from our metaanalyses for all G6PD allelic variants reported in Online Mendelian Inheritance in Man (OMIM) ${ }^{24}$ Only four of these were polymorphic: G6PD-Canton, G6PDKaiping, G6PD-RFLP (rs2230037), and G6PD-Viangchan (rs137852327; online supplementary table 8). G6PD variants identified through previous GWAS were either monomorphic in our Asian individuals ${ }^{356}$ or not associated with HbA1c ( $p>0.02) .{ }^{25}$ G6PD-Viangchan, a missense variant reported in Cambodians, Thais, Laotians, and Malaysia's Malays, ${ }^{26}{ }^{27}$ showed suggestive association with HbA1c in SiMES Malay men $\left(\mathrm{MAF}=0.9 \%\right.$, effect ${ }_{\text {men }}=-0.64 \%, 95 \%$ CI -0.92 to $-0.36,-7.0 \mathrm{mmol} / \mathrm{mol}, 95 \%$ CI -10.1 to -3.9 , $\left.\mathrm{p}=7.21 \times 10^{-5}\right)$. Due to a low imputation info score, G6PDViangchan could not be confirmed in Living Biobank, and

a meta-analysis with exome sequence data did not reach genome-wide significance $\left(\mathrm{MAF}=0.8 \%\right.$, effect $_{\text {men }}=-0.62 \%$, $95 \%$ CI -0.85 to $-0.39,-6.8 \mathrm{mmol} / \mathrm{mol}, 95 \%$ CI -9.3 to $\left.-4.3, \mathrm{p}=3.44 \times 10^{-6}\right)$.

No association with glucose measurements at G6PD locus Both G6PD-Canton and G6PD-Kaiping variants were not associated with $\mathrm{FG} / \mathrm{RG}$ ( $\mathrm{p}>0.04$; online supplementary table 9). Effect estimates on HbA1c did not attenuate on adjusting for $\mathrm{FG} / \mathrm{RG}$ (online supplementary table 10 ), suggesting that these genetic effects were glucose independent.

\section{Relationship between HbA1c and FG by G6PD-Canton genotypes}

Compared with women, the difference in mean HbA1c by G6PD-Canton genotypes was more pronounced in men (figure 1A,B and online supplementary table 11). We generated scatterplots of HbAlc on FG. HbAlc increased with FG in male non-carriers (Pearson's $r=0.37$ ) but not in carriers (Pearson's $\mathrm{r}=-0.33$, online supplementary tables 12 and 13, figure 1C,D). To determine whether the genetic effect varied by FG, we performed interaction analyses within men and women separately, regressing $\mathrm{HbA1c}$ on FG, G6PD-Canton genotype, and the interaction term, FG*G6PD-Canton. Genetic effects differed by sex $\left(\mathrm{p}=3.32 \times 10^{-5}\right)$, indicating that the HbA1clowering effect of G6PD-Canton increased with higher FG. The relationship between HbA1c and FG did not differ by genotype in women $(\mathrm{p}=0.29)$. Due to low allele count $(\mathrm{AC}=13)$, similar analyses were not performed for G6PD-Kaiping.

\section{Proportion of pre-diabetes/undiagnosed diabetes by G6PD- Canton genotype classes}

In 2566 individuals with no prior diagnosis of diabetes or medication use, and both HbAlc and FG measured, 738 men $(69.0 \%)$ and 1035 women $(69.1 \%)$ were classified as having normoglycemia (FG $<100 \mathrm{mg}$ / $\mathrm{dL}(5.6 \mathrm{mmol} / \mathrm{L})$ and HbAlc $<5.7 \%$ (39 mmol/ $\mathrm{mol})$. The remaining had pre-diabetes/undiagnosed diabetes defined by $\mathrm{FG} \geq 100 \mathrm{mg} / \mathrm{dL}(5.6 \mathrm{mmol} / \mathrm{L})$ or $\mathrm{HbA} 1 \mathrm{c} \geq 5.7 \%$ (39 mmol/mol), ADA criteria). In men, the proportion of FG-defined pre-diabetes/undiagnosed diabetes was similar between carriers and noncarriers $(p=0.21)$ but the proportion of HbAlc-defined diabetes/undiagnosed diabetes was lower in carriers (307 non-carriers $(30 \%)$ and 1 carrier $(5 \%), \mathrm{p}=0.03$; figure 2A,B and online supplementary table 14). In women, there were no significant differences $(p>0.1)$ (figure 2C,D and online supplementary table 14). When using WHO criteria for pre-diabetes/diabetes, none of the male carriers of G6PD-Canton were classified as having pre-diabetes/diabetes.

\section{DISCUSSION}

While there have been extensive discovery efforts on the genetics of HbAlc in European ancestry, recent 

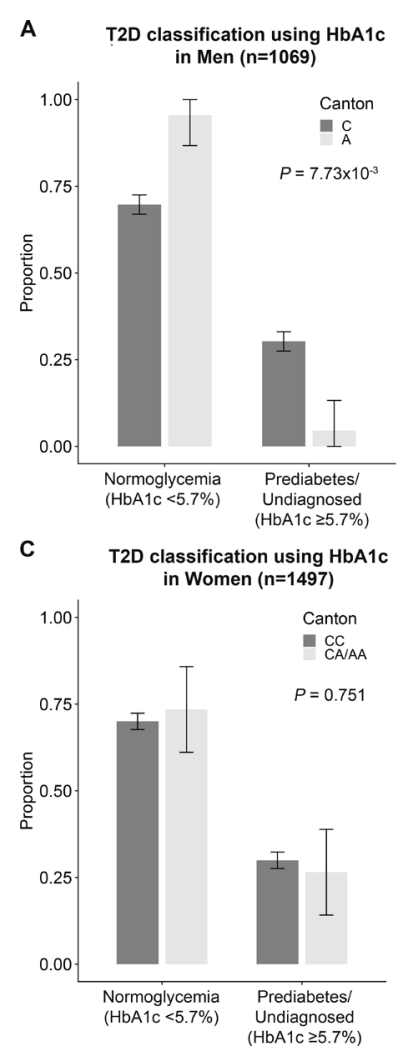

Figure 2 Proportions of individuals by pre-diabetes/ diabetes status using American Diabetes Association (ADA) guidelines for fasting glucose $\geq 100 \mathrm{mg} / \mathrm{dL}(5.6 \mathrm{mmol} / \mathrm{L})$ and $\mathrm{HbA} 1 \mathrm{c} \geq 5.7 \%$ (39 $\mathrm{mmol} / \mathrm{mol})$ in men and women separately. (A) HbA1c thresholds in men. (B) Fasting glucose threshold in men. (C) HbA1c thresholds in women. (D) Fasting glucose threshold in women. $\mathrm{P}$ values are from Fisher's exact test comparing normoglycemia with pre-diabetes/undiagnosed diabetes. FG, fasting glucose; HbA1c, hemoglobin A1c; T2D, type 2 diabetes.

studies assessing the impact of genetics on the diagnostic accuracy of HbAlc in Asians are limited. Here, we undertook a large-scale association analysis of variants on the X chromosome in 10005 EAS and 2051 SAS from eight Asian studies to identify Asian-specific G6PD variants associated with HbAlc. We identified associations of two distinct low-frequency $G 6 P D$ variants, G6PD-Canton and G6PD-Kaiping, which lowered HbA1c independently of glycemia. Additionally, we showed that diabetes or pre-diabetes would be underdiagnosed in carriers of these $G 6 P D$ variants if screened only by HbA1c without confirmation by direct glucose measurements. These findings highlight the importance of genetic discovery efforts to uncover ancestryspecific variants with direct clinical implications on diagnostic tests which are routinely performed around the world.

G6PD deficiency confers a relative resistance against Plasmodium falciparum malaria. ${ }^{28}$ This balancing selection pressure leads to the accumulation of G6PD alleles in malaria-endemic countries. Howes et al used geostatistical modeling to map the prevalence of G6PD deficiency in malaria-endemic countries and showed that G6PD deficiency is spatially heterogenous across these countries. ${ }^{10}$ While occurring globally, most people with G6PD deficiency reside in Asia. ${ }^{9}$ G6PD-Canton $(\mathrm{MAF}=2.2 \%)$ and G6PD-Kaiping ( $\mathrm{MAF}=1.6 \%$ ) have been classified by WHO as class II variants that are associated with severe acute hemolysis due to low enzymatic activity $(<10 \%) .{ }^{29}$

By contrast, the common African-specific G6PD variant (rs1050828, G6PD-Asahi, MAF $=11 \%$ ) has been classified by $\mathrm{WHO}$ as a class III variant with partial enzymatic activity (10\%-60\% enzymatic activity) that is generally only associated with intermittent hemolysis. ${ }^{29}$ Among these three variants, G6PD-Kaiping has the lowest frequency and the largest HbAlc-lowering effect. In Singapore, a country with three major Asian ethnicities (Chinese, Malay and Indian), we identified three distinct low-frequency $G 6 P D$ variants that differed in their effects on HbAlc: G6PD-Canton in Chinese, G6PD-Kaiping in Chinese, ${ }^{18}$ G6PD-Viangchan in Malays, ${ }^{26}$ and none in Indians. These three variants are specific to Asians and monomorphic in other ancestries.

Prevailing diagnostic thresholds for HbA1c are conservative relative to $\mathrm{FG}^{2}{ }^{2}$ Our findings suggest that the diagnostic sensitivity of HbAlc for diabetes or pre-diabetes is likely poorer in carriers of these G6PD variants than non-carriers, supporting the use of FG over HbA1c for diabetes screening of individuals with known G6PD deficiency. We recognize that not every country with G6PD deficiency has universal screening for the disease. In countries without universal screening, asymptomatic carriers are unlikely aware of their carrier status. Moreover, despite HbAlc having several advantages compared with FG (eg, measurement in the non-fasting state, capturing of overall glycemia, and lower biological variability), NGSP-certified HbAlc testing may not be readily accessible in some parts of the world and is generally more costly more than glucose measurements. Thus, the use of both FG and HbAlc in combination for diabetes diagnosis is paramount in individuals with unknown carrier status, especially when screening ethnically diverse populations that include people with African, Arabian or Asian ancestry.

We observed a slightly lower than expected effect in females (sex heterogeneity $\mathrm{p}_{\text {Canton }}=0.00051, \mathrm{p}_{\text {Kaiping }}=0.071$ ). In an additive model, we would have expected the difference in HbA1c between hemizygous males and those without the variant to be double the difference between heterozygous females and those without the variant due to random $\mathrm{X}$ inactivation. Heterogeneity of genetic effects by sex could be due to X linked dosage compensation or skewed inactivation in heterozygous females. ${ }^{30}$ It is also possible that females with one normal copy of the gene maintain adequate enzymatic activity and do not have any or only have subclinical disease, in keeping with an X linked recessive genetic disorder. Alternatively, other non-glycemic factors (eg, iron status) could have contributed to heterogeneity of effects by sex.

Our study has several strengths. This study represents one of the largest genetic discovery efforts of HbAlc for the $\mathrm{X}$ chromosome in multiethnic Asian ancestry. 
Singapore is a relatively young multiethnic country with immigrants from China, India, and the Malaysia and Indonesia archipelagos. While our study samples are predominantly Chinese, and may not represent the full complement of genetic effects on HbAlc in the geographically diverse Asian populations, the association of these low-frequency G6PD variants with $\mathrm{HbA1c}$ helped inform the unique genetic architecture of HbA1c in individuals from EAS and SAS ancestries. These G6PD variants likely cause clinically meaningful reductions in HbA1c, highlighting the potential impact of genetics on the diagnostic classification of diabetes by HbA1c.

We acknowledge limitations. We did not distinguish type 1 and type 2 diabetes, though HbA1c is used to estimate glycemia in both types of diabetes. As continuous glucose monitoring data were not available, we were unable to estimate the concordance between measured HbAlc and HbAlc estimated from continuous glucose monitoring average glucose. ${ }^{31} 32$ Nevertheless, continuous glucose monitoring is not routinely used to detect pre-diabetes or diabetes, and so comparing the diagnostic classification by HbA1c to FG is more aligned with current screening practices. As we did not have measures of G6PD enzymatic activity or other blood traits in our study, we were unable to formally test for mediating mechanisms underlying the relationship between G6PDCanton and G6PD-Kaiping with HbA1c. Nevertheless, both of these G6PD variants have been previously classified by the WHO as class II variants that cause severe G6PD deficiency $(<10 \%$ residual enzymatic levels and intermittent hemolysis), in keeping with shortened erythrocytic life span as the mechanism involved in lowering HbA1c. ${ }^{9} 102933$ While we were able to demonstrate the effect of G6PD-Canton on reclassification of diabetes and pre-diabetes status, larger samples are needed to perform a similar analysis for G6PD-Kaiping. As this investigation focused solely on the $\mathrm{X}$ chromosome, we recognize that variation in the rest of the genome was not examined and sequence data would have strengthened the discovery of HbAlc-related variants in the low allele frequency spectrum, especially in these populations. The limited exome sequence data in smaller sample set were either included in the meta-analysis as independent samples or used to verify the ChrX imputation that we had undertaken using array genotypes. However, previously reported HbAlcassociated loci in Asian ancestry, alone or in combination, have effect sizes several times smaller than $G 6 P D$ variants, and likely play a much smaller role in the overall contribution of genetics to underdiagnosis of diabetes by HbA1c.

In addition to diagnostic testing, HbA1c is routinely used in the management of diabetes to assess glycemic control, disease progression, prediction of diabetesrelated complications, and response to treatment. We posit that HbA1c remains a reasonable biomarker to assess change in glycemia over time for both carriers and non-carriers. However, in a treat-to-A1C-target paradigm, physicians may be falsely reassured by low HbAlc values and undertreat or delay treatment escalation in carriers. These carriers may have higher overall glycemia and higher rates of diabetes-related complications compared with non-carriers. We note that the HbAlc-lowering effect of G6PD-Canton variant seemed to increase with higher FG within the non-diabetic range in men, implying that the HbAlc-lowering effect of variant could be greater in the diabetic range. Considering the small sample size, this association needs to be interpreted with caution. Future work could focus on replicating this finding in independent samples with both sexes. Further research is also required to determine whether genetic effects of $G 6 P D$ variants on $\mathrm{HbA1c}$ are larger in people with diabetes, and whether rates of diabetes-related complications, delayed diagnoses or undertreatment differ by genotype in realworld clinical settings.

In this study, we identified low-frequency Asian-specific $G 6 P D$ variants associated with non-glycemic reductions in HbA1c. Carriers of these G6PD variants could have delayed diagnosis or underdetection of diabetes. Given the genetic diversity of $G 6 P D$ variants globally, and the growing use of $\mathrm{HbAl} \mathrm{c}$ as a diagnostic test worldwide, individual G6PD genotype could be considered in precision medicine approaches to diabetes screening in ethnically diverse populations, even if the proportion of affected individuals is small. Both HbA1c and FG ought to be used in combination for diabetes diagnosis in carriers of these $G 6 P D$ variants to avoid potential disparities in care.

\section{Author affiliations}

${ }^{1}$ Department of Medicine, Massachusetts General Hospital, Boston, Massachusetts, USA

${ }^{2}$ Harvard Medical School, Boston, Massachusetts, USA

${ }^{3}$ Saw Swee Hock School of Public Health, National University of Singapore,

Singapore

${ }^{4}$ Department of Epidemiology and Biostatistics, Key Laboratory for Environment and Health, School of Public Health, Tongji Medical College, Huazhong University of Science and Technology, Wuhan, Hubei, China

${ }^{5}$ Genome Institute of Singapore, Agency for Science, Technology and Research, Singapore

${ }^{6}$ Department of Paediatrics, Yong Loo Lin School of Medicine, National University of Singapore, Singapore

${ }^{7}$ Khoo Teck Puat-National University Children's Medical Institute, National University Health System, Singapore

${ }^{8}$ Health Services and Systems Research, Duke NUS Medical School, Singapore ${ }^{9}$ Division of Cancer Control and Population Sciences, UPMC Hillman Cancer Center, University of Pittsburgh, Pittsburgh, Pennsylvania, USA

${ }^{10}$ Department of Epidemiology, Graduate School of Public Health, University of Pittsburgh, Pittsburgh, Pennsylvania, USA

${ }^{11}$ Department of Ophthalmology, Medical Faculty Mannheim, University of Heidelberg, Heidelberg, Baden-Württemberg, Germany

${ }^{12}$ Ophthalmology and Visual Sciences Key Laboratory, Beijing Institute of Ophthalmology, Beijing Tongren Hospital, Capital Medical University, Beijing, China

${ }^{13}$ Beijing Key Laboratory of Intraocular Tumor Diagnosis and Treatment, Beijing Tongren Eye Center, Beijing Tongren Hospital, Capital Medical University, Beijing, China

${ }^{14}$ Department of Medicine, Yong Loo Lin School of Medicine, National University of Singapore, Singapore

${ }^{15}$ Genetics, Merck Sharp and Dohme IA, Kenilworth, New Jersey, USA

${ }^{16}$ Singapore Eye Research Institute, Singapore National Eye Centre, Singapore

${ }^{17}$ Ophthalmology and Visual Sciences Academic Clinical Program (Eye ACP), DukeNUS Medical School, Singapore 
Acknowledgements The authors thank all the investigators, staff members, and study participants for their contributions to all the participating studies. The authors are grateful to the NUH Tissue Repository for tissue archival services and the Genome Institute of Singapore, Agency for Science, Technology and Research, Singapore for genotyping (SCES, SiMES, SINDI, MEC, SP2, Living Biobank and SCHS-MI).

Contributors AL, VJYL, and XS conceptualized, planned and conducted the study, interpreted the results, and wrote the manuscript. VJYL, CW, RD, and JFC performed the data analyses. CKH, RMvD, WPK, JMY, JBJ, YXW, WBW, JL, DFR, TYW, and CYC were involved in the study design, sample collection and data generation (phenotype, genotype). All authors reviewed and approved the manuscript for submission. XS and AL had full access to all the data in the study and had final responsibility for the decision to submit for publication. XS and AL are the guarantors of the work.

Funding The Diabetic Cohort (DC), Multi-Ethnic Cohort (MEC) and Living Biobank was supported by grants from the Ministry of Health, Singapore, the National University of Singapore and the National University Health System, Singapore. In addition, genotyping for Living Biobank was funded by the Agency for Science, Technology and Research, Singapore, and Merck Sharp \& Dohme Corp., Whitehouse Station, NJ, USA. The Singapore Prospective Study Program (SP2) were supported by the individual research grant and clinician scientist award schemes from the National Medical Research Council (NMRC) and the Biomedical Research Council (BMRC) of Singapore. The Singapore Chinese Eye Study (SCES) and the Singapore Malay Eye Study (SiMES) are supported by the National Medical Research Council (NMRC), Singapore (grants 0796/2003, 1176/2008, 1149/2008, STaR/0003/2008, 1249/2010, CG/SERI/2010, CIRG/1371/2013, and CIRG/1417/2015), and Biomedical Research Council (BMRC), Singapore (08/1/35/19/550 and 09/1/35/19/616). The Singapore Chinese Health Study was supported by the National Institutes of Health, USA (R01 CA144034 and UM1 CA182876), the nested case-control study of myocardial infarction by the Singapore National Medical Research Council (NMRC 1270/2010) and genotyping by the HUJ-CREATE Programme of the National Research Foundation, Singapore (Project No 370062002). The Beijing Eye Study (BES) was funded by the National Natural Science Foundation of China (NSFC, No 81170890 and 81570835).

Competing interests None declared.

Patient consent for publication Not required

Ethics approval This study was approved by the National University of Singapore International Review Board (08-013) and conducted according to the Declaration of Helsinki.

Provenance and peer review Not commissioned; externally peer reviewed.

Data availability statement Summary-level statistics will be publicly available at http://blog.nus.edu.sg/agen/summary-statistics/hba1c-chrx-2020/

Open access This is an open access article distributed in accordance with the Creative Commons Attribution Non Commercial (CC BY-NC 4.0) license, which permits others to distribute, remix, adapt, build upon this work non-commercially, and license their derivative works on different terms, provided the original work is properly cited, appropriate credit is given, any changes made indicated, and the use is non-commercial. See: http://creativecommons.org/licenses/by-nc/4.0/.

ORCID iD

Xueling Sim http://orcid.org/0000-0002-1233-7642

\section{REFERENCES}

1 Mortensen HB, Christophersen C. Glucosylation of human haemoglobin $A$ in red blood cells studied in vitro. Kinetics of the formation and dissociation of haemoglobin A1c. Clin Chim Acta 1983;134:317-26.

2 American Diabetes Association. 2. Classification and Diagnosis of Diabetes: Standards of Medical Care in Diabetes-2018. Diabetes Care 2018;41:S13-27.

3 Wheeler E, Leong A, Liu C-T, et al. Impact of common genetic determinants of hemoglobin A1c on type 2 diabetes risk and diagnosis in ancestrally diverse populations: a transethnic genomewide meta-analysis. PLoS Med 2017;14:e1002383.

4 Leong A, Wheeler E. Genetics of HbA1c: a case study in clinical translation. Curr Opin Genet Dev 2018;50:79-85.
5 Moon J-Y, Louie TL, Jain D, et al. A Genome-Wide Association Study Identifies Blood Disorder-Related Variants Influencing Hemoglobin $A_{1 c}$ With Implications for Glycemic Status in U.S. Hispanics/Latinos. Diabetes Care 2019;42:1784-91.

6 Sarnowski C, Leong A, Raffield LM, et al. Impact of rare and common genetic variants on diabetes diagnosis by hemoglobin $A 1 C$ in Multi-Ancestry cohorts: the Trans-Omics for precision medicine program. Am J Hum Genet 2019;105:706-18.

7 Motulsky AG, Stamatoyannopoulos G. Clinical implications of glucose-6-phosphate dehydrogenase deficiency. Ann Intern Med 1966;65:1329-34.

8 Nkhoma ET, Poole C, Vannappagari V, et al. The global prevalence of glucose-6-phosphate dehydrogenase deficiency: a systematic review and meta-analysis. Blood Cells Mol Dis 2009;42:267-78.

9 Howes RE, Piel FB, Patil AP, et al. G6Pd deficiency prevalence and estimates of affected populations in malaria endemic countries: a geostatistical model-based MAP. PLoS Med 2012;9:e1001339.

10 Howes RE, Dewi M, Piel FB, et al. Spatial distribution of G6PD deficiency variants across malaria-endemic regions. Malar $J$ 2013;12:418.

11 WHO. Use of glycated haemoglobin ( $H b A 1 c)$ in the diagnosis of diabetes mellitus: abbreviated report of a who consultation, 2011.

12 Auton A, Brooks LD, Durbin RM, et al. A global reference for human genetic variation. Nature 2015;526:68-74.

13 Delaneau O, Zagury J-F, Marchini J. Improved whole-chromosome phasing for disease and population genetic studies. Nat Methods 2013;10:5-6.

14 Das S, Forer L, Schönherr S, et al. Next-Generation genotype imputation service and methods. Nat Genet 2016;48:1284-7.

15 Fuchsberger C, Flannick J, Teslovich TM, et al. The genetic architecture of type 2 diabetes. Nature 2016;536:41-7.

16 Flannick J, Mercader JM, Fuchsberger C, et al. Exome sequencing of 20,791 cases of type 2 diabetes and 24,440 controls. Nature 2019;570:71-6.

17 Dou J, Sun B, Sim X, et al. Estimation of kinship coefficient in structured and admixed populations using sparse sequencing data. PLoS Genet 2017;13:e1007021.

18 Quak SH, Saha N, Tay JS. Glucose-6-Phosphate dehydrogenase deficiency in Singapore. Ann Acad Med Singapore 1996;25:45-8.

19 Zhan X, Hu Y, Li B, et al. RVTESTS: an efficient and comprehensive tool for rare variant association analysis using sequence data. Bioinformatics 2016;32:1423-6.

20 Willer CJ, Li Y, Abecasis GR. Metal: fast and efficient meta-analysis of genomewide association scans. Bioinformatics 2010;26:2190-1.

21 Pruim RJ, Welch RP, Sanna S, et al. LocusZoom: regional visualization of genome-wide association scan results. Bioinformatics 2010;26:2336-7.

22 Liu X, Jian X, Boerwinkle E. dbNSFP v2.0: a database of human nonsynonymous SNVs and their functional predictions and annotations. Hum Mutat 2013;34:E2393-402.

23 McLaren W, Gil L, Hunt SE, et al. The Ensembl variant effect predictor. Genome Biol 2016;17:122.

24 Hamosh A, Scott AF, Amberger JS, et al. Online Mendelian inheritance in man (OMIM), a knowledgebase of human genes and genetic disorders. Nucleic Acids Res 2005;33:D514-7.

25 De Vita G, Alcalay M, Sampietro M, et al. Two point mutations are responsible for G6PD polymorphism in Sardinia. Am J Hum Genet 1989;44:233-40.

26 Ainoon O, Yu YH, Amir Muhriz AL, et al. Glucose-6-Phosphate dehydrogenase (G6PD) variants in Malaysian Malays. Hum Mutat 2003;21:101.

27 Bancone G, Menard D, Khim N, et al. Molecular characterization and mapping of glucose-6-phosphate dehydrogenase (G6PD) mutations in the greater Mekong subregion. Malar J 2019;18:20.

28 Luzzatto L, Arese P, Favism AP. Favism and glucose-6-phosphate dehydrogenase deficiency. N Engl J Med 2018;378:60-71.

29 WHO. Glucose-6-Phosphate dehydrogenase deficiency. who Working group. Bull World Health Organ 1989;67:601-11.

30 Sidorenko J, Kassam I, Kemper KE, et al. The effect of X-linked dosage compensation on complex trait variation. Nat Commun 2019;10:3009.

31 Malka R, Nathan DM, Higgins JM. Mechanistic modeling of hemoglobin glycation and red blood cell kinetics enables personalized diabetes monitoring. Sci Transl Med 2016;8:359ra130.

32 Bergenstal RM, Beck RW, Close KL, et al. Glucose management indicator (GMI): a new term for estimating A1c from continuous glucose monitoring. Diabetes Care 2018:41:2275-80.

33 Yoshida A, Beutler E, Motulsky AG. Human glucose-6-phosphate dehydrogenase variants. Bull World Health Organ 1971;45:243-53. 